\title{
Effect of vertical velocity gradient on ground motion in a sediment-filled basin due to incident $S V$ wave
}

\author{
Yanbin Wang ${ }^{1}$, Hiroshi Takenaka ${ }^{1}$, and Takashi Furumura ${ }^{2}$ \\ ${ }^{1}$ Department of Earth and Planetary Sciences, Kyushu University, Hakozaki 6-10-1, Fukuoka 812-8581, Japan \\ ${ }^{2}$ Faculty of Education, Hokkaido University of Education, Midorigaoka 2-34-1, Iwamizawa 068-0835, Japan
}

(Received July 17, 1998; Revised September 7, 1999; Accepted November 22, 1999)

\begin{abstract}
The natural sedimental deposits in basins show strong vertical heterogeneity in their material parameters. The aim of this paper is to investigate the effects of such vertical heterogeneity, especially vertical velocity gradient, inside basin on the seismic ground motion through the parametric study on the response of a twodimensional semi-cylindrical sediment-filled basin to a vertical incidence of plane $S V$ wave using the pseudospectral method. This numerical study has tried to find the effects caused by vertical velocity gradient through the use of synthetic seismograms, wavefield snapshots and surface amplitude distribution. Simulation results clearly demonstrate the detailed character of wave propagation phenomena in basins with vertical velocity gradient, which produces characteristic amplification pattern of the surface motion caused mainly by the generation of strong Rayleigh wave induced at the basin edge associated with large lateral velocity change across the basin edge. Amplification pattern at the surface strongly depends on both the vertical velocity gradient in the basin and the predominant frequency of the incident wave. Although similar phenomena on wave propagation and surface motion found in previous studies for homogeneous basin models have also been observed in this study, it has been found that the vertical velocity gradient enhances such phenomena. The results suggest that it is important to represent the vertical velocity profiles accurately when we construct a structural model for realistic modelling of ground motion.
\end{abstract}

\section{Introduction}

Damage caused by disastrous earthquake has been observed to concentrate in some restricted areas, but not simply attenuate with the distance from the epicenter. This has been supposed to be related to the local site effects due to surface topography and sediment characteristics just below the surface, which cause drastic amplification of seismic motion at the surface, as observed in the 1985 Michoacan, Mexico (Anderson et al., 1986), 1994 Northridge (Gao et al., 1996) and 1995 Hyogo-ken Nanbu (Kawase, 1996) earthquakes. Experimental observations on sediment-filled valleys have also shown that the ground motion varies with the location inside the valley and the property of sediment deposits (e.g., King and Tucker, 1984). Since many major cities are built on top of sediment-filled basins, it is quite important to investigate the effects of sediment characteristics on ground motion from the point view of disaster mitigation.

Through numerical studies, it has been found that the ground motion on top of sedimentary basin is influenced by the shape of basin boundary (deep or shallow basin), impedance contrast between sediments and bedrock, incident angles of wave, and dissipation behavior of sediments. In realistic geological structures, the natural sedimental deposit is observed to be inhomogeneous in space, especially in vertical profile. In this study, we

Copy right $\odot$ The Society of Geomagnetism and Earth, Planetary and Space Sciences (SGEPSS); The Seismological Society of Japan; The Volcanological Society of Japan; The Geodetic Society of Japan; The Japanese Society for Planetary Sciences. investigate the phenomena related to the vertical inhomogenity of the sedimental deposit inside basin. Effects of vertical velocity gradient on the wave amplification in sedimentary basin have been studied for several models with different methods mainly for $\mathrm{SH}$ wave incidence (Bard and Gariel, 1986; Benites and Aki, 1994). In this paper, the phenomena caused by vertical velocity gradient in a $2 \mathrm{D}$ sediment-filled basin is investigated in case of incident $S V$ wave by numerical simulation using the pseudospectral method. Since the $S V$ wave impinging on the material boundaries such as a basin/bedrock interface and the free surface causes strong $S V$-to- $P$ conversion at the interface and in consequence strong Rayleigh wave is produced at the surface, the ground amplification pattern produced by the $S V$-wave incidence should be more complicated than that from the $\mathrm{SH}$ wave.

Numerical studies on the ground motion in sedimentfilled basin have been carried out during last three decades (Takenaka, 1993; Hisada and Yamamoto, 1996). Recent developments in computation technology and the numerical simulation algorithm are making it available to apply 3D simulations of ground motion to realistic structures (e.g., Hisada et al., 1993; Graves, 1998; Furumura et al., 1998). By using realistic 3D models, the effects of various influence factors on ground motion are mixed in a complex manner, which precludes profound understanding of the effect of each factor. For fully understanding the effect of a specific influence factor, it 
is preferred to use a rather simple or idealized $2 \mathrm{D}$ model. For example, in order to fully understand the "basin-edge effect", Kawase et al. (1998) used a 2D homogeneous rectangular basin embedded in a homogeneous half space subject to plane $S V$-wave incidence instead of a realistic 3D model in which the "basin-edge effect" is mixed with other effects. By using this model, they clearly identified the generation mechanism of the "basin-edge effect", extracted the edge-induced waves and could quantitatively estimate that these edge-induced waves give at least 50 $\mathrm{cm} / \mathrm{s}$ additional amplitude in terms of the peak ground velocity inside the damage belt during Hyogo-ken Nanbu earthquake. In this paper, we use a simple 2D semicylindrical shaped basin embedded in homogeneous half space as the model. This model makes possible investigation focusing on the effects of vertical velocity gradient within sedimentary basin. The results will be investigated by means of the synthetic seismograms of the surface motion, the snapshots of the wave propagation, and the surface maximum displacement distribution. Incident waves with different predominant frequencies are used in the calculation, in order to find the possible frequency effect of input signal on the ground amplification.

\section{Method and Models}

The equations of momentum conservation for a twodimensional medium in the absence of body force term are given by

$$
\begin{gathered}
\rho \ddot{u}=\frac{\partial \sigma_{x x}}{\partial x}+\frac{\partial \sigma_{x z}}{\partial z}, \\
\rho \ddot{w}=\frac{\partial \sigma_{x z}}{\partial x}+\frac{\partial \sigma_{z z}}{\partial z},
\end{gathered}
$$

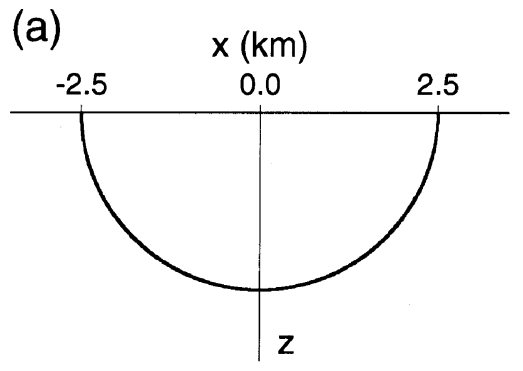

(c)

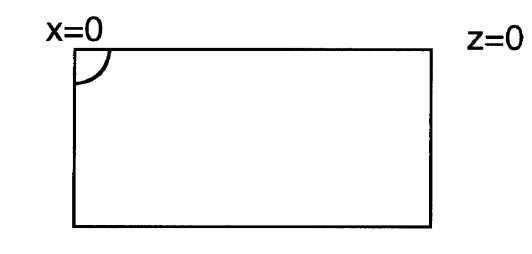

where $\sigma_{x x}, \sigma_{z z}$ and $\sigma_{x z}$ are the stress components, $\ddot{u}$ and $\ddot{w}$ are the second time derivatives of displacements (i.e., accelerations) in the $x$ and $z$ directions, $\rho$ is the density.

In isotropic elastic media, the stress components are given by

$$
\begin{aligned}
& \sigma_{x x}=\lambda\left(e_{x x}+e_{z z}\right)+2 \mu e_{x x}, \\
& \sigma_{z z}=\lambda\left(e_{x x}+e_{z z}\right)+2 \mu e_{z z}, \\
& \sigma_{x z}=2 \mu e_{x z},
\end{aligned}
$$

where $e_{x x}, e_{z z}$ and $e_{x z}$ are the strain components defined as

$$
\begin{aligned}
& e_{x x}=\frac{\partial u}{\partial x}, \\
& e_{z z}=\frac{\partial w}{\partial z}, \\
& e_{x z}=\frac{1}{2}\left(\frac{\partial w}{\partial x}+\frac{\partial u}{\partial z}\right) .
\end{aligned}
$$

For solving the equations, the numerical modeling scheme by the pseudospectral method (e.g., Kosloff and Baysal, 1982; Kosloff et al., 1984; Furumura et al., 1996) is adopted. In this scheme, the spatial derivatives in Eqs. (1) and (3) are approximated by using the fast Fourier transform, and time derivatives are calculated with the second order finite differencing. The validity and accuracy of this method (e.g., the numerical dispersion and reflections from the artificial boundaries) have been already discussed in details in published papers (e.g.,

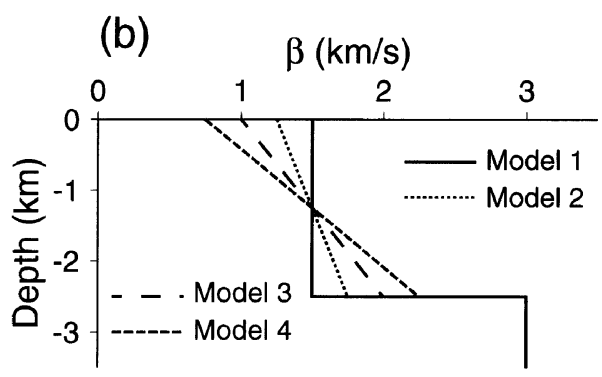

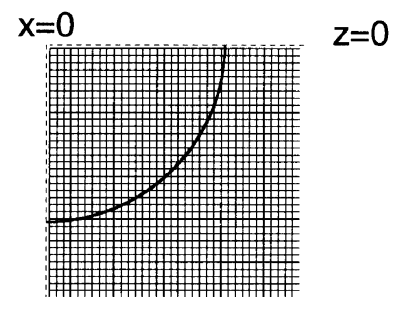

Fig. 1. Model configuration and velocity distribution in depth for the four models used in the simulation. (a) A sediment-filled semi-cylindrical basin of radius $a=2.5 \mathrm{~km}$ embedded in a homogeneous half space. (b) The velocity distribution for the four models. (c) The half domain and part of the mesh used in the simulations, dash line is the boundary of the real model. 
Kosloff and Baysal, 1982; Fornberg, 1987; Daudt et al., 1989; Furumura et al., 1998). In order to check the validity and accuracy of our pseudospectral code used in this paper, we have compared the synthetic seismograms calculated by pseudospectral method with those obtained using the discrete wave number method for a half space model. The comparison is shown in Appendix.

Seismic disturbance from the plane $S V$-wave incidence is incorporated in the calculation through the use of initial condition of displacement and stress components distribution in the two-dimensional coordinates.

We consider a semi-cylindrical basin of radius $a=2.5$ $\mathrm{km}$ which is embedded in a homogeneous half space as depicted in Fig. 1(a). The reason for choosing such a deep basin model is mainly because it allows the vertical velocity gradient to be well represented and moreover seismic wave propagation character to be clearly displayed in the snapshot. The homogeneous half space bedrock has $P$ - and $S$-wave velocities of $\alpha=5.2 \mathrm{~km} / \mathrm{s}$ and $\beta=3.0 \mathrm{~km} /$ $\mathrm{s}$, respectively, and density of $\rho=2.6 \mathrm{~g} / \mathrm{cm}^{3}$. Inside the basin, one homogeneous case and three inhomogeneous cases with velocity gradient of $0.2,0.4$ and $0.6 \mathrm{~s}^{-1}$ referred as Model 1, 2, 3 and 4, respectively, are selected. The shear wave velocity for the inhomogeneous cases increases linearly from the surface to the bottom of the basin, and the average velocity of them is equal to that of the homogeneous case. The compressional wave velocity in the basin is also modified in the same way as the shear wave velocity, by using constant density of $\rho=2.0 \mathrm{~g} / \mathrm{cm}^{3}$ for the sediment throughout the model. Figure 1(b) displays the shear wave velocity distribution in vertical direction for the four models. The size of the models used in the simulation is $12.8 \mathrm{~km}$ deep and $25.6 \mathrm{~km}$ long. The grid points used for horizontal and vertical directions are 256 and 128 , respectively, and the spatial mesh size is $0.1 \mathrm{~km}$ in both directions. Since geometry of the model and input $S V$ plane wave is symmetric about the vertical axis, only right half of the model is discretized and used in calculation as shown in Fig. 1(c) to reduce the computer memory and the CPU time, and the results of the half-domain modeling can be extended to the other half by symmetric and antisymmetric extension to get the whole wavefield (Takenaka et al., 1999). The time increment used in the simulation is $0.005 \mathrm{~s}$ to satisfy the stability condition, and 2000 time steps is used for the synthetic seismograms of $10 \mathrm{~s}$ duration. Absorbing boundaries (Cerjan et al., 1985) of 20 grid points wide are set at the bottom and the right side of the model to minimize the wraparound effect. The free surface (top dash line in Fig. 1(c)) is placed half grid size above the surface of discretized model. Stress components on grid points half grid size above the free surface in the air are set to be zeros. Vertical incident $S V$ plane wave of Ricker wavelet type with unit peak amplitude in time is employed as the input signal. The predominant frequency of the input wave (the central frequency of the Ricker wavelet) is measured in terms of the non-dimensional frequency $\eta=2 a / \lambda$, where the wavelength $\lambda$ is taken as the value in the homogeneous basement. In the simulation, five values of $\eta, 0.75,1.0,2.0,3.0$ and 4.0 are used. Observation points are set from the center of the basin to
$5.0 \mathrm{~km}$ at intervals of $0.1 \mathrm{~km}$ along the model surface. In the following figures, the observation site location $(x / a)$ is measured from the basin center and scaled to the radius of the basin.

\section{Synthetic Seismograms and Snapshots 3.1 Homogeneous basin}

3.1.1 Wavefield snapshots Figure 2 illustrates the snapshots of wavefield at ten time steps in Model 1 for incident wave with the predominant frequency $\eta=2.0$. This non-dimensional frequency corresponds to $1.20 \mathrm{~Hz}$, and the wavelength of input signal equals the radius of the basin. The snapshots are represented by the $P$-wave and $S V$-wave contributions, which are shown in red and blue, respectively. Contributions of $P$ and $S V$ waves are calculated from the divergence and curl of the wavefield,

$$
P:\left|\frac{\partial u}{\partial x}+\frac{\partial w}{\partial z}\right|, \quad S V:\left|\frac{\partial w}{\partial x}-\frac{\partial u}{\partial z}\right|
$$

Since attention is made especially for the wavefield inside the basin, the area around basin with $6.4 \mathrm{~km}$ in horizontal and $3.2 \mathrm{~km}$ in vertical direction is only shown in Fig. 2. The snapshots give a clear image of the wave propagation inside and around the basin. The image at $t=1.2 \mathrm{~s}$ shows the refracted $S V$ wave through the basin boundary with a bending wave front inside the basin due to slow wave propagation velocity inside the semi-cylindrical basin in contrast with higher $S V$ wave velocity outside the basin. Ahead of the refracted $S V$ wave is the red area that is the refracted $P$ wave generated by conversion from the $S V$ wave at the boundary, which has minor amplitude at the center area of the basin because no conversion occurs from vertical wave incidence. At time $t=2.0 \mathrm{~s}$, the $S V$ wave arrives at the basin surface and reflected $S V$ and $P$ wave from the surface appears. Near both edge areas, the edge-generated Rayleigh waves appear along a very shallow zone of the basin surface, which just follow the interference between $S V$ and $P$ waves. The snapshot at time $t=2.3 \mathrm{~s}$ shows the evolution of the main phase observed at the surface in early time, which is the large blue areas beside the basin center. The reflected $S V$ and $P$ waves propagating downward inside the basin can also be clearly identified in this snapshot. At $t=2.8 \mathrm{~s}$, the Rayleigh waves arrive near the basin center. The reflected $S V$ and $P$ waves from the surface propagating downward are clearly displayed in the snapshot at $t=3.3 \mathrm{~s}$. Snapshot at $t=4.4$ s illustrates the reflected $S V$ wave from the surface which escapes into half space bedrock, while the large-amplitude Rayleigh waves remain inside the basin and propagate in a shallow zone along the basin surface. They propagate to the other side of the basin with large energy and the maximum amplitude at the surface. At $t=5.0 \mathrm{~s}$, the Rayleigh waves propagate to the areas near basin edges. Some reflections of down-going $S V$ wave from the basin bottom can also be found in the snapshots at $t=6.0 \mathrm{~s}, 7.0$ $\mathrm{s}$ and $8.0 \mathrm{~s}$. The seismic wave energy trapped in the basin is gradually weakened through the multiple reflections within the basin and accordingly the seismic energy escapes 


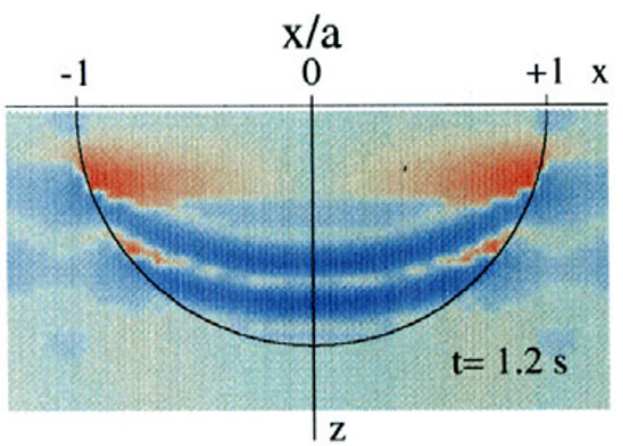

$R$
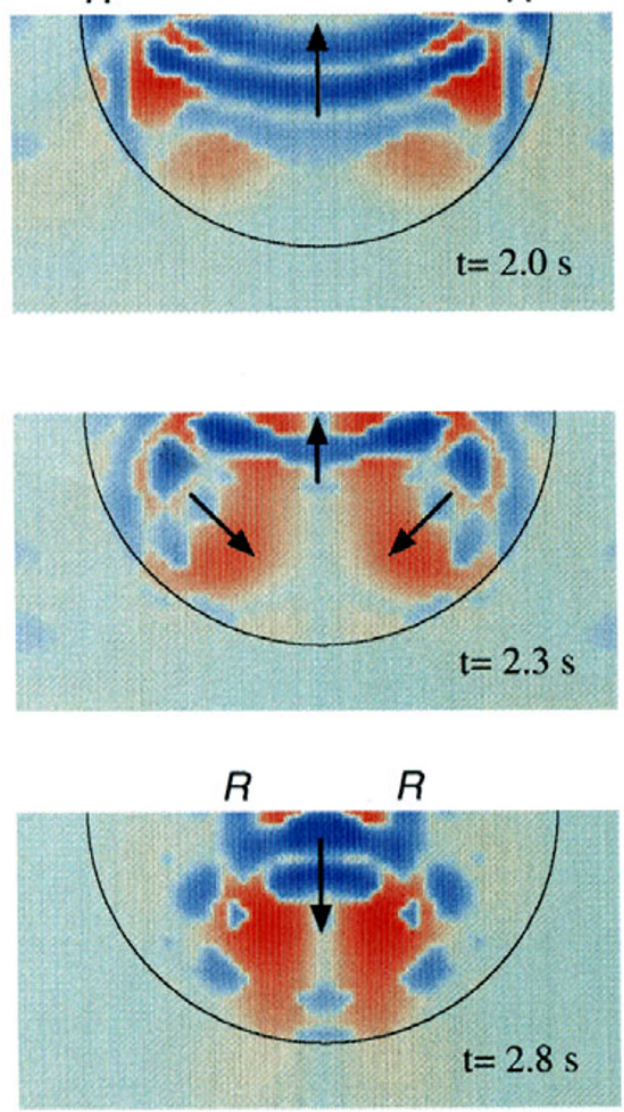

\section{$R$}

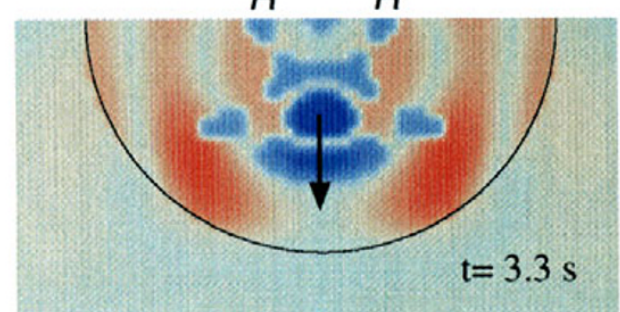

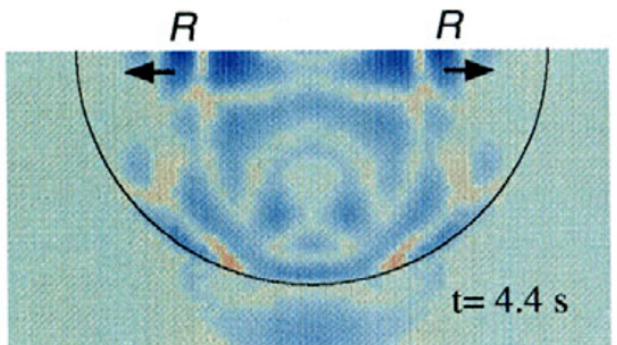

$R$
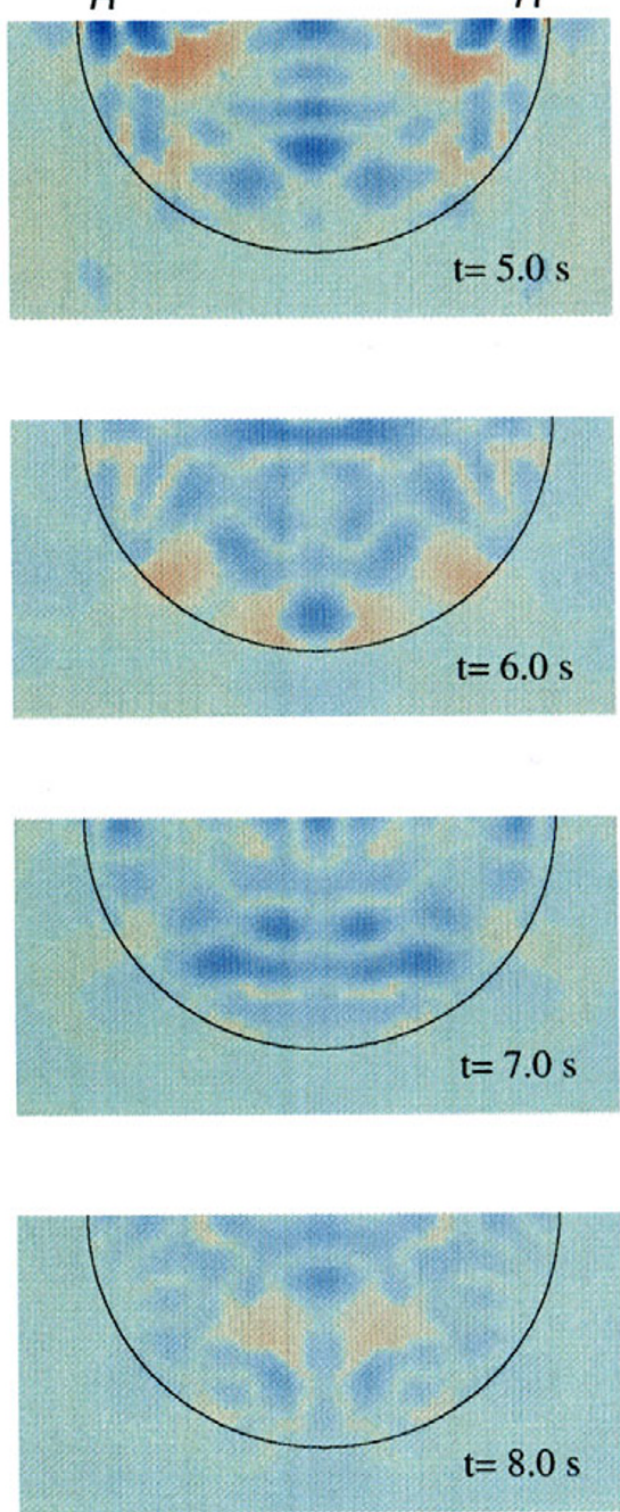

Fig. 2. Snapshots at ten time steps showing the evolution of observed phases and the propagation of waves inside and around basin for homogeneous Model 1. The red and blue areas represent $P$ and $S V$ motion respectively. Intenser color corresponds to larger energy. Arrows indicate the direction of wave propagation, and " $\mathrm{R}$ " denotes the Rayleigh wave. The scale of snapshots is $6.4 \mathrm{~km}$ in horizontal and $3.2 \mathrm{~km}$ in vertical direction.

outside the basin. At later time of $t=6.0 \mathrm{~s}, 7.0 \mathrm{~s}$ and 8.0 $\mathrm{s}$, the wavefield inside the basin and the surface motion becomes very weak.

3.1.2 Synthetic seismograms Figure 3 shows the horizontal and vertical components of synthetic seismograms for 100 observation points along the surface for the same model as in Fig. 2. The amplitudes of seismograms shown in this figure are normalized by the same factor for all the observation points.

Figure 3(a) is the horizontal component $(u)$ of motion along the surface, $x / a$ between -1 and 1 indicates the range along basin surface, outside of them are surface of 
(a) Model 1

$\mathrm{u}$

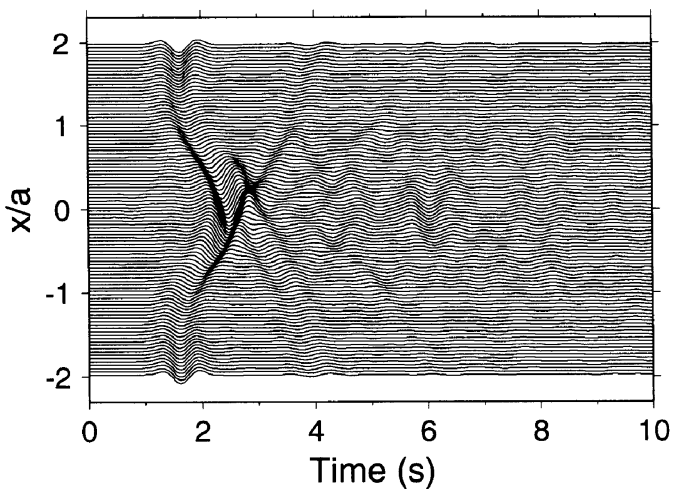

(b) Model 1

W

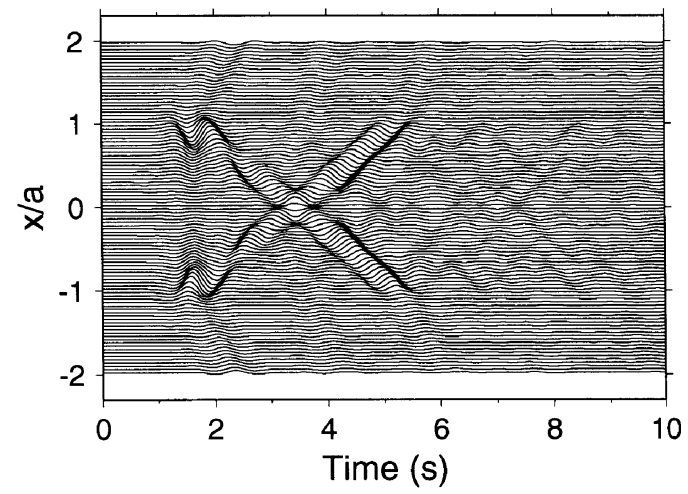

Fig. 3. Synthetic seismograms at 100 observation stations along the surface of Model 1 with incident wave with the predominant frequency $\eta=2.0$. The vertical axis represents the location of the observation sites, where -1 and 1 are the positions of the basin edges. (a) is the horizontal component, (b) is the vertical component. All traces are normalized by the same factor.

homogeneous half space and $-1,1$ represent the basin edges. On the surface of homogeneous half space, the incident wave and the reflection from the surface are observed between $1.0 \mathrm{~s}$ and $2.0 \mathrm{~s}$. Inside the basin, the first phase observed starts from both edges with minor amplitude and propagates along the surface. Amplitude of this phase increases toward the center of basin and reaches the maximum value at the center. It can be clearly identified that this phase propagates between $1.5 \mathrm{~s}$ and $4.0 \mathrm{~s}$, and contributes mostly to the early time of horizontal ground motion inside the basin. It is generated when the $S V$ wave reaches the basin surface, meanwhile the reflected $S V$ and reflected $P$ waves are generated at the basin surface. Interference of these three waves causes this phase that has the largest horizontal amplitude and propagates along the basin surface. The apparent velocity of this phase is $3.0 \mathrm{~km} / \mathrm{s}$, which is just the $P$-wave velocity inside the basin. It transmits to the homogeneous half space when reaches the basin boundary, and is then slightly reflected back to the basin. At around $6.0 \mathrm{~s}$ and $8.0 \mathrm{~s}$, reflections of the $S V$ waves from bottom boundary of the basin can be identified around the central area of the basin with decreasing amplitude.

Figure 3(b) shows the vertical component $(w)$ of the surface motion at the same observation points as in Fig. 3(a). The first phase observed is the refracted $P$ wave generated when the incident $S V$ wave has reached the basin boundary. It appears around $1.5 \mathrm{~s}$ with minor amplitude. Amplitude of the refracted $P$ wave increases from the center to both edges of the basin since the incident angle of $S V$ wave increases toward both edges. The maximum vertical amplitude is observed at both edge areas between $1.0 \mathrm{~s}$ and $2.0 \mathrm{~s}$. At the edge areas, the incident $S V$ wave reaches the basin boundary with very large angle of incidence, and therefore the refracted $S V$ and $P$ waves propagate nearly vertically between the surface and the basin boundary. This narrow zone between the basin surface and the basin boundary causes constructive interference of multiple refracted/reflected $S V$ and $P$ waves within this restricted area, and the interference generates the maximum vertical motion inside the basin. The constructive interference at the edge areas gradually grows to the Rayleigh surface waves at the areas near edges, which is the most prominent phase as clearly seen in the vertical component starting from around $2.0 \mathrm{~s}$. The Rayleigh waves propagate laterally along the surface of the basin and are reflected at the basin boundary, and contribute mainly to the middle and later time of the ground motion inside the basin.

Common features mentioned above for seismograms on the basin surface of Model 1 are also observed for incident wave with other frequencies, although they are not displayed here because of space limitation.

We have examined the features of wave propagation and surface motion inside the basin for homogeneous model. In the following subsection, we consider the basin models with vertical velocity gradient.

\subsection{Inhomogeneous basin}

Figure 4 displays the seismograms for inhomogeneous Model 2, 3 and 4 with incident wave with the same predominant frequency $\eta=2.0$ as in Fig. 3. The main phases mentioned above for the homogeneous case can also be observed in both horizontal and vertical components in Fig. 4 but show different wave characters. Figure 4(a) is the results for Model 2 with velocity gradient rate of 0.2 $\mathrm{s}^{-1}$. In the horizontal component, the interference of refracted/reflected $S V$ and $P$ waves is still dominant in the early time, but the maximum horizontal amplitude does not appear at the center as in Model 1 and shifts from center area to both sides of the center. The duration of this phase is longer and the phase velocity is lower than that in Model 1. At later time of the seismograms, more phases are generated inside the basin, especially in the areas near to the edges, which cannot be found in the homogeneous case. In the vertical component, refracted $P$ wave and interference at edge areas appear also as the first phase. The basin-edge generated Rayleigh waves are also dominant at middle and later time of the seismograms but have larger amplitude and lower phase velocity as compared with those in Model 1. The surface wave propagation time from one edge to the other is about $t=2.0 \mathrm{~s}$ to $5.8 \mathrm{~s}$, while that for the homogeneous case is 

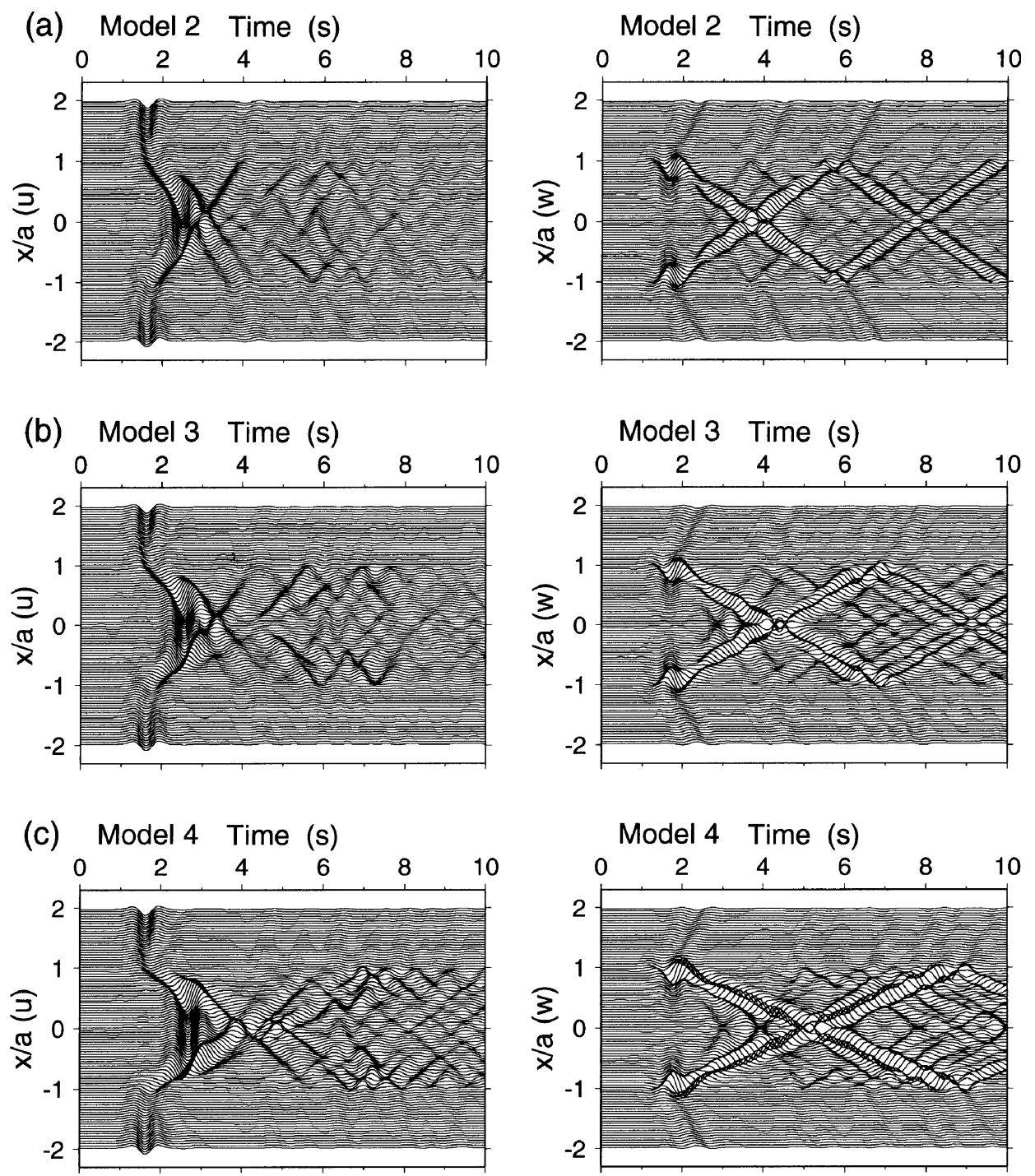

Fig. 4. Synthetic seismograms at 100 observation stations along the surface of inhomogeneous models with incident wave with the predominant frequency $\eta=2.0$. (a), (b) and (c) are the results for Model 2, 3, and 4, respectively. The left show horizontal components, the right show vertical components. All traces are normalized by the same factor as that for seismograms in Fig. 3.

about $t=2.0 \mathrm{~s}$ to $5.0 \mathrm{~s}$. The reflection of Rayleigh waves from the basin boundary is much stronger than that in Model 1. At later time of the seismograms, more phases are also generated especially near the edge areas as observed also in the horizontal component.

For Model 3 with much larger velocity gradient rate of $0.4 \mathrm{~s}^{-1}$ (Fig. 4(b)) and Model 4 of strong velocity gradient rate of $0.6 \mathrm{~s}^{-1}$ (Fig. 4(c)), the similar characteristics as seen in Model 2 are severely enhanced. The differences can be identified among them are as follows: In both horizontal and vertical components, the maximum amplitude shifts towards the edges; the interference phase and the edge generated Rayleigh waves have much longer duration, lower phase velocity and larger amplitude; more later phases are generated with larger amplitudes with increasing of the velocity gradient.

Figure 5 shows the snapshots of wavefield at the same ten time steps for the incident wave with the same predominant frequency as in Fig. 2 but for Model 4 of the largest vertical velocity gradient. Comparing Figs. 2 and
5 , we see that the vertical velocity gradient has strong effect on the wavefield inside the basin, the velocity gradient effect is rather obvious especially for the surface motion within an upper shallow zone of the basin. Comparing with the homogeneous model (Model 1) in Fig. 2, we find the changing of the curvature of refracted and reflected wave fronts, which is caused by the velocity gradient (snapshots of $t=1.2 \mathrm{~s}$ to $3.3 \mathrm{~s}$ ). The edgegenerated Rayleigh waves can also be clearly seen with larger amplitude than that in Fig. 2. In snapshots at $t=2.0$ $\mathrm{s}$ to $3.3 \mathrm{~s}$, the refracted and reflected waves are "trapped" at the upper shallow zone with large amplitude inside the basin due to the very low velocity in the upper zone. This cannot be observed for the homogeneous basin. At middle to later time of $t=5.0 \mathrm{~s}$ to $8.0 \mathrm{~s}$, the motion along the shallow surface zone is very strong, which is caused by later phases of both $P$ and $S V$ waves. At later time of $t=7.0 \mathrm{~s}$ and $8.0 \mathrm{~s}$, we see that the strong surface motion is concentrated around the edge areas of the basin. The motion inside the basin and along the basin surface is 

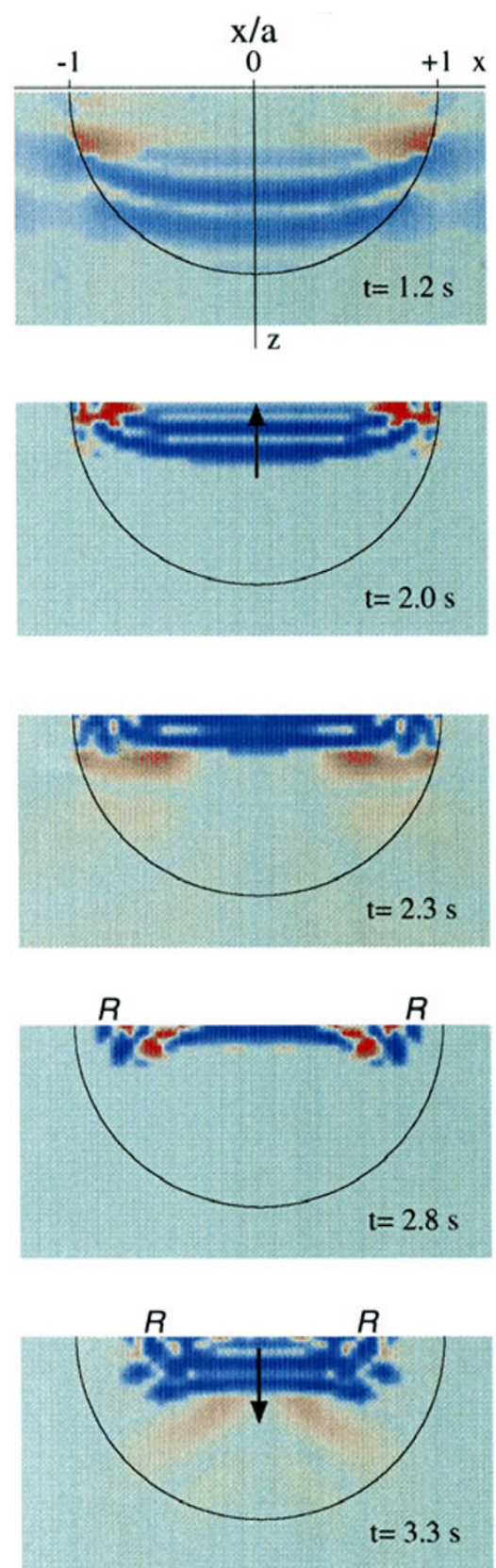
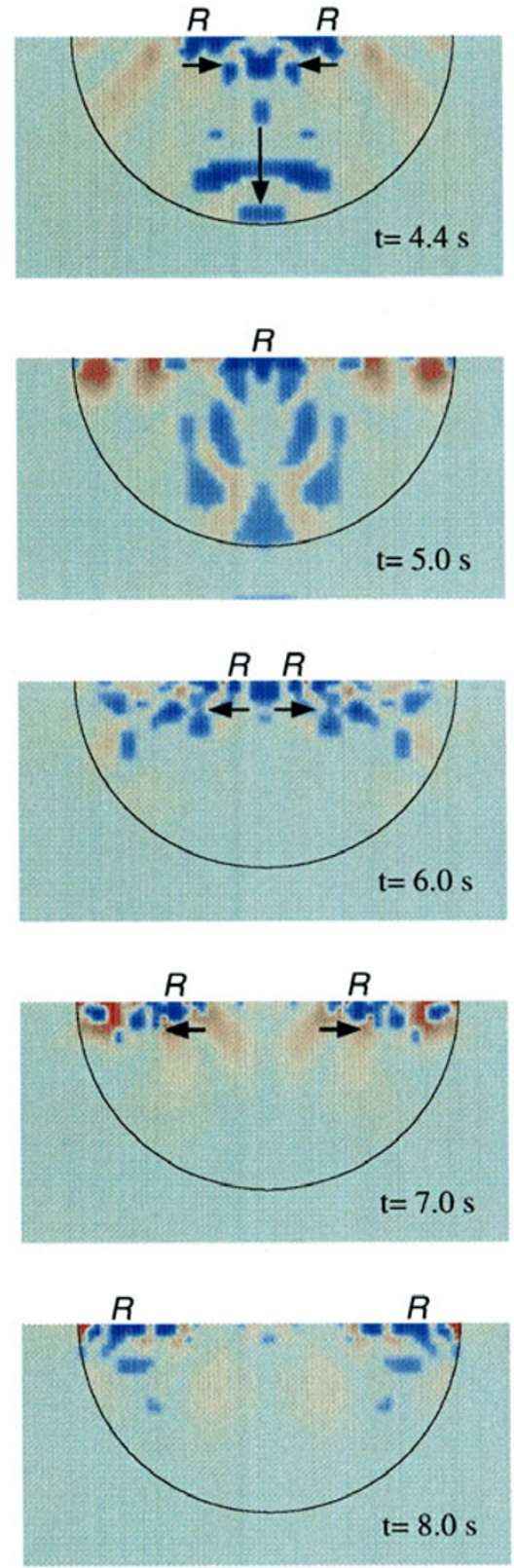

Fig. 5. Snapshots at ten time steps showing the evolution of observed phases and the propagation of waves inside and around basin for inhomogeneous Model 4. The red and blue areas represent $P$ and $S V$ motion respectively. Intenser color corresponds to larger energy. Arrows indicate the direction of wave propagation, and " $\mathrm{R}$ " denotes the Rayleigh wave. The scale of snapshots is $6.4 \mathrm{~km}$ in horizontal and $3.2 \mathrm{~km}$ in vertical direction.

much stronger at later time of $t=6.0 \mathrm{~s}$ to $8.0 \mathrm{~s}$ as compared with the results of the homogeneous model in Fig. 2.

The similar phenomena mentioned above have also been observed among the four models for incident wave with other predominant frequencies.

When the non-dimensional frequency $\eta$ equals 0.75 and 1.0 , the corresponding wavelengths of the incident wave are respectively $6.7 \mathrm{~km}$ and $5.0 \mathrm{~km}$ which are much larger than the radius of the basin. The Rayleigh surface waves are not excited for these frequencies. The Rayleigh surface waves excited near edge area are prominent for $\eta=2.0$, 3.0, 4.0 especially in the vertical component seismograms as shown in Figs. 3 and 4, because the corresponding wavelengths $(2.5 \mathrm{~km}, 1.7 \mathrm{~km}$ and $1.25 \mathrm{~km})$ are smaller than the radius of the basin. We calculated the theoretical dispersion curves of Rayleigh waves for the four basin velocity models by using the matrix method for multilayered media (Haskell, 1953). For the calculations, we divide the media in the basin from surface to the bottom into 25 layers with constant thickness of $0.1 \mathrm{~km}$. The calculated dispersion curves are shown in Fig. 6 . Since we keep the average velocity in the basin at the depth of the half radius constant for the four models, increasing velocity gradient from Model 1 to Model 4 leads to decreasing the velocity near the surface and increasing the velocity near the bottom of the basin. The dispersion curves are nearly the same for frequencies lower than $0.35 \mathrm{~Hz}$, while the differences among the four 


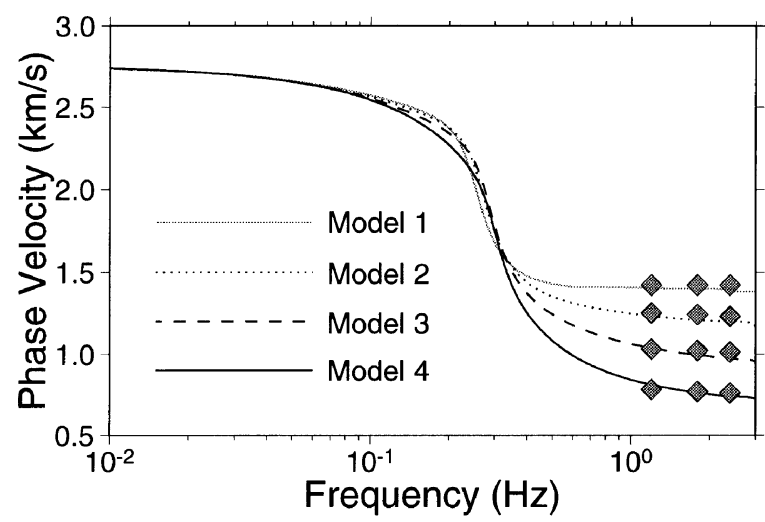

Fig. 6. Comparison of the observed phase velocities of Rayleigh waves with the theoretical dispersion curves for the four models. Four lines represent the theoretical dispersion curves for the four models, and diamonds are the observed phase velocities.

dispersion curves appear for the frequencies higher than $0.35 \mathrm{~Hz}$, which are caused by the differences of shallow layer velocities. In our simulations, the frequencies used for input signals are $0.45,0.60,1.20,1.80$ and $2.40 \mathrm{~Hz}$ which allow us to identify the differences between the Rayleigh waves excited in the four models.

We measured the phase velocity of Rayleigh waves for all the four models with $\eta$ of 2.0, 3.0 and 4.0, and found it is nearly the same for these frequency values for the same model. The phase velocities measured for Model 1, 2,3 and 4 are around $1.4,1.2,1.0$ and $0.8 \mathrm{~km} / \mathrm{s}$, respectively. We plotted the observed phase velocities in Fig. 6, which agree very well with the theoretical dispersion curves. The phase velocities of the Rayleigh waves decrease with increasing of the velocity gradient inside the basin. Since the low frequency Rayleigh waves are not excited in such small basins, we cannot observe the well dispersive Rayleigh waves in the synthetic seismograms.

The changing of vertical velocity distribution causes the above-mentioned changing of characteristics of various phases. For homogeneous model, the $S V$ wave transmitting into the basin focuses at the central area of the basin and the maximum horizontal displacement appears there. In case of gradient models, the curvature of the wavefront of the $S V$ wave refracted at the basin boundary becomes smaller at central area and larger at edges. The focusing of refracted $S V$ wave spreads away from the center to both sides of the center, and the distance of the spreading away to the central area increases with increasing of the velocity gradient, so that the maximum horizontal displacement is observed away from the center. Secondary generated Rayleigh waves become stronger with increasing of the velocity gradient because the impedance contrast becomes larger around the edge areas and the multiple reflection/ refraction at edge areas becomes stronger, then the generated Rayleigh waves have larger amplitude. The shallow part of the basin has lower seismic wave velocity with increasing of the velocity gradient, so that the Rayleigh waves have lower phase velocity and longer duration for larger gradient model. Less energy can leak to outside of the basin for greater gradient case and in consequence seismic energy remains inside the basin for long time. Since the velocity difference between the shallow zone and the deep zone inside the basin and that between the basin and the bedrock at shallow part become larger with increasing of the velocity gradient, the scattering of wavefield inside the basin becomes stronger. The scattered waves appear as later phases on the basin surface as observed in the seismograms and snapshots for gradient cases, especially near the edge areas.

\section{The Surface Amplitude \\ 4.1 Effect of vertical velocity gradient}

To investigate the effect of velocity gradient on the surface amplification, we show in Fig. 7 the maximum horizontal and vertical displacement values at the surface observation points for the four models. Figures from top to bottom are the results for the four models with incident wave of different frequency. Four curves from the bottom to the top in each figure represent results of Model 1, 2, 3 and 4 with increasing vertical velocity gradient as labeled at the right side of Fig. 7(a). For the sake of clear comparison and display, the lines are shifted by an offset of two units between each other.

Figure 7(a) displays the distribution of the horizontal and vertical maximum displacements for the incident wave with the predominant frequency of $\eta=0.75$. The maximum horizontal amplitude appears at the center of the basin for homogeneous model (Model 1-the bottom curve) and the models with velocity gradient rate of $0.2 \mathrm{~s}^{-1}$ and $0.4 \mathrm{~s}^{-1}$, and shifts to both sides of the center for model with velocity gradient rate of $0.6 \mathrm{~s}^{-1}$ (Model 4- the top curve). The maximum amplitude on the top of the half space bedrock is 2.0, which is twice the amplitude of the incident wave. For the maximum vertical displacement, all of the four models show common characteristics that the maximum amplitude appears at the two edge areas. Inside the basin, the surface vertical amplitude increases with increasing of the velocity gradient. On the surface of homogeneous half space, the maximum vertical amplitude is about 0.5 which is the amplitude of the wave propagating from basin outward to the half space. For both horizontal and vertical components, the pattern of displacement distribution at the basin surface becomes complicated with increasing of the velocity gradient.

Figure 7(b) is the results for incident wave with the predominant frequency of $\eta=1.0$. For the horizontal component, the maximum amplitude is also observed apart from the center to both sides of the center with increasing of the velocity gradient. For the vertical component, the maximum amplitude also appears at the edge areas. For incident wave with predominant frequency of $\eta=2.0,3.0$, and 4.0, the results shown in Figs. 7(c) to (e) represent the similar features as mentioned above. In Figs. 7(d) and (e), large local maximum amplitudes appear at the center and the edge areas of the basin, which are caused by the later phases generated inside the basin for the model with velocity gradient of $0.6 \mathrm{~s}^{-1}$.

For incident waves with different frequencies, the four models have similar distribution patterns of the maximum surface displacement to each other, but the distribution 


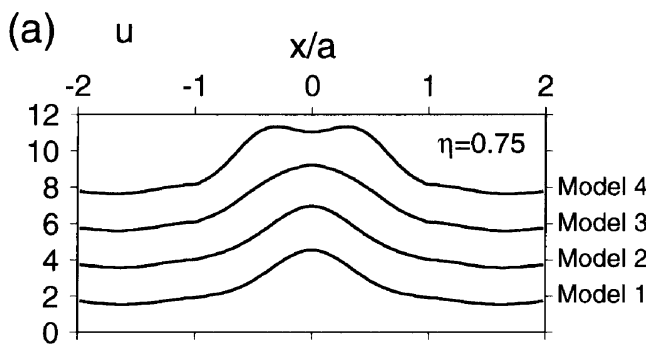

(b)

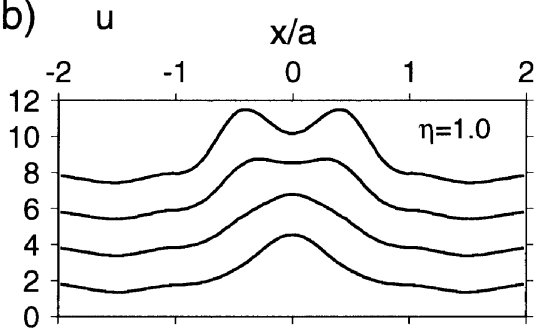

(c) $u$

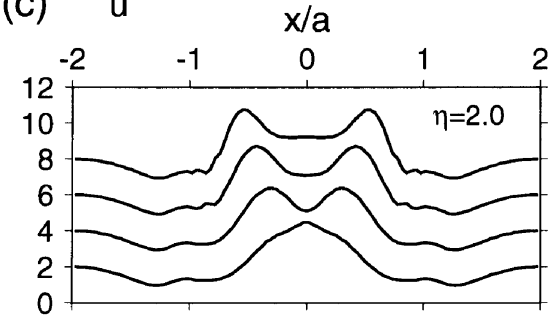

(d) $u$

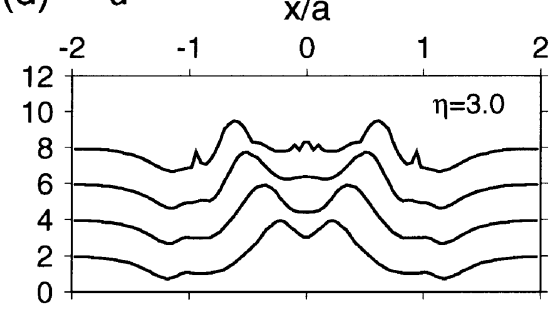

(e) $u$

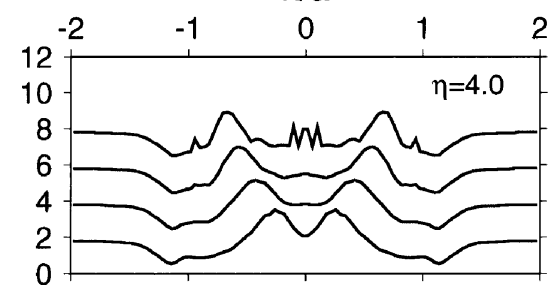

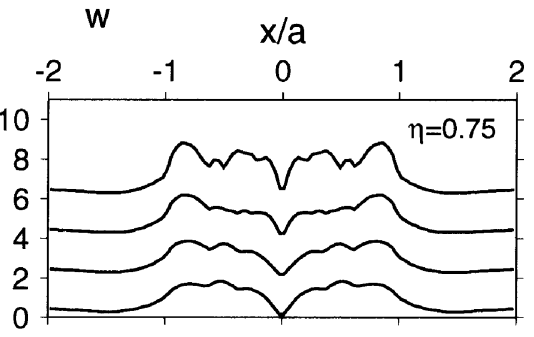
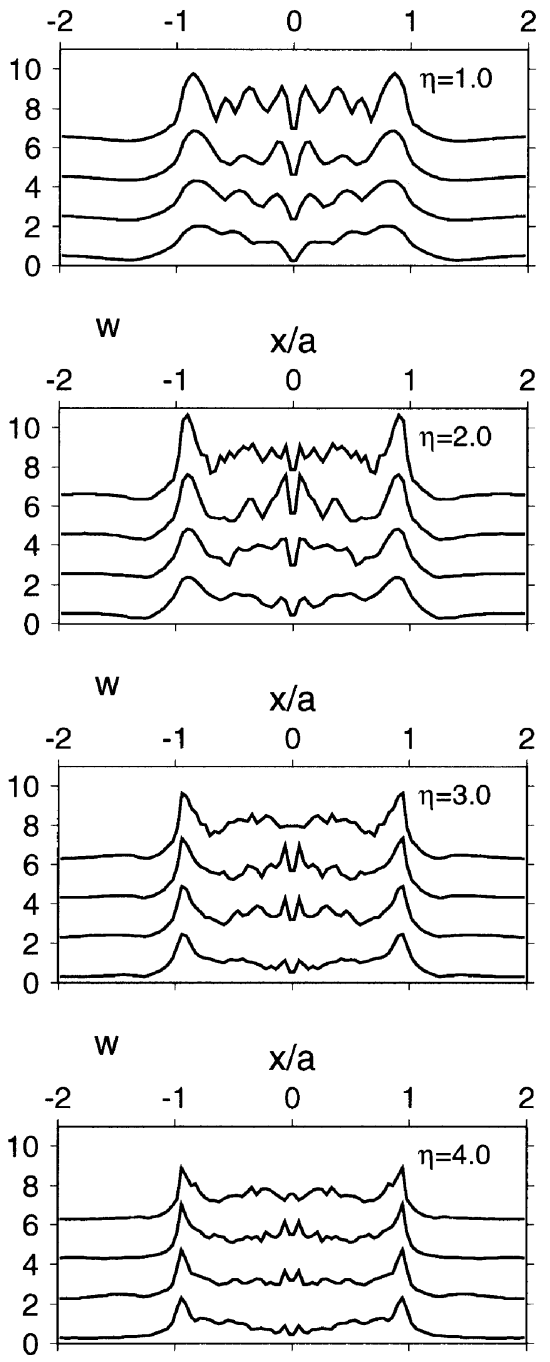

Fig. 7. Distribution of the maximum surface amplitude for the four models and each frequency. From (a) to (e) are the results for predominant frequency $\eta$ of $0.75,1.0,2.0,3.0$ and 4.0. Left and right figures show the horizontal and vertical components, respectively. In each figure, from the bottom to the top are the results for Model 1,2,3, and 4. The four curves are shifted upward by an offset of two units for comparison and clear display.

pattern tends to be more complex and peak amplification is more localized with increasing of the velocity gradient. Along the surface of the basin and the outside areas near edges, both the horizontal and vertical motions vary drastically from place to place, with prominent local maximum. The basin surface and nearby areas undergo strong differential horizontal and vertical motions during the wave propagation process in this simulation.

\subsection{Effect of frequency of input signal}

To focus on effect of frequency of the input signal, we rearrange the data in Fig. 7 and display them in Fig. 8. In each figure of Fig. 8, the five curves from top to bottom represent the results for the same model with input wave of different predominant frequency as labeled at the right side of Fig. 8(a). For the horizontal component, the peak of the maximum amplitude distribution locates at the basin center for low frequency input as shown in the left column of Fig. 8. With increasing of the frequency, the peak separates into twin peaks which locate beside the basin center. For the homogeneous basin (Model 1) the peak value of the maximum horizontal surface displacement is similar each to each frequency as shown 
(a)

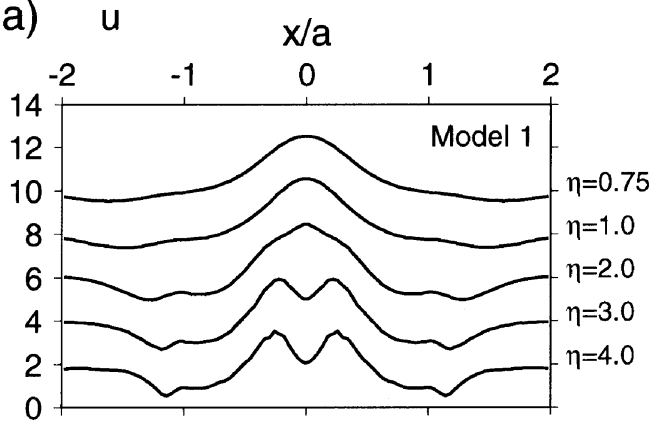

(b)

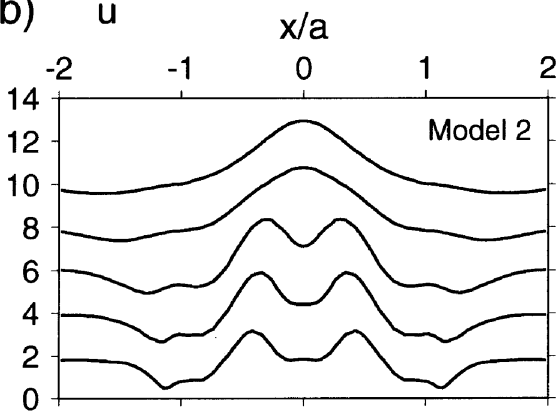

(c) $u$

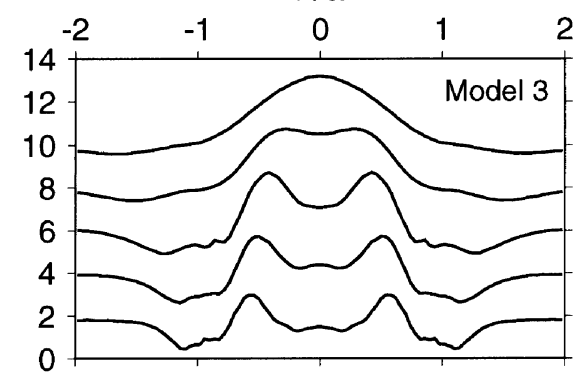

(d) $u$

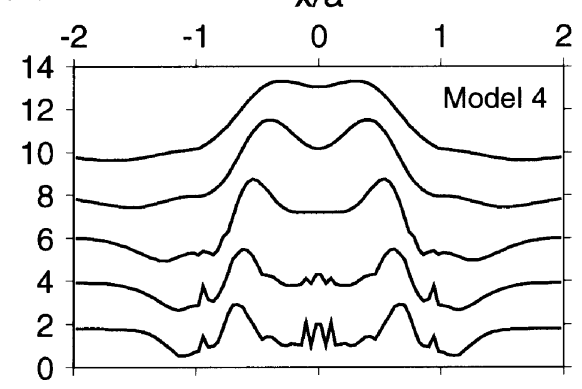

w

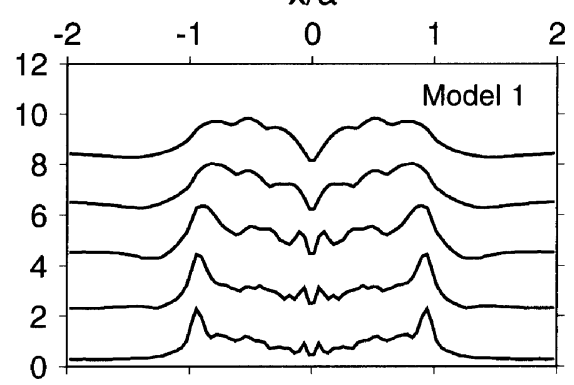

W

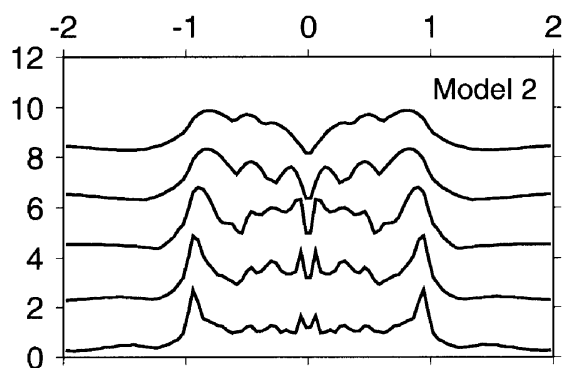

W

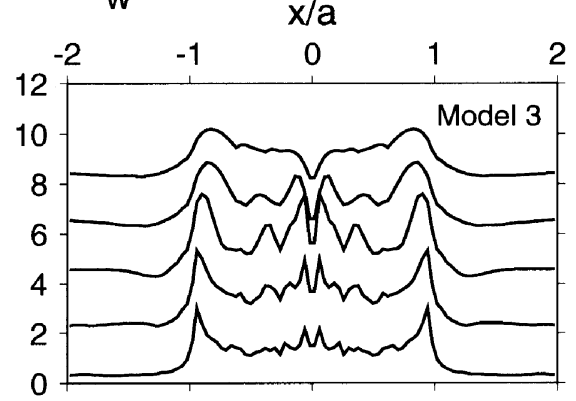

W

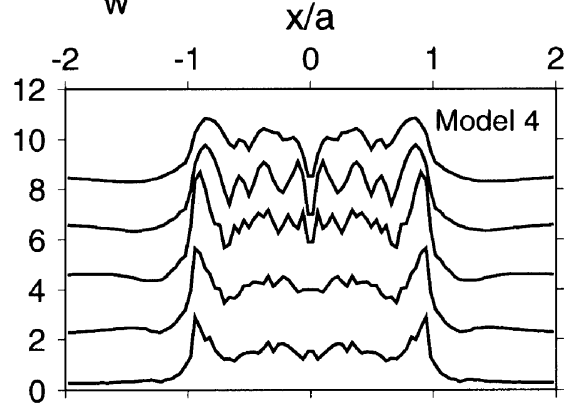

Fig. 8. Distribution of the maximum surface amplitude for the four models and each frequency. Results for Model 1, 2, 3 and 4 are shown in (a) to (d). Left and right figures are the horizontal and vertical components, respectively. In each figure, from the top to the bottom are the results for $\eta$ of $0.75,1.0,2.0,3.0$ and 4.0. The five curves are shifted by an offset of two units between each other.

in Fig. 8(a), while for inhomogeneous basin models the peak value reduces with increasing of the frequency as observed clearly in left figures of Figs. 8(b) to (d). For the vertical component, peaks of the maximum surface amplitude locate near the basin edges, and approaching the edges become sharp with increasing of the frequency. The peak values for $\eta$ of 0.75 and 4.0 are smaller than those for $\eta$ of 1.0 and 3.0, and the maximum peak value is observed when $\eta$ equals 2.0. The difference of the maximum surface amplitude between the basin edges and inside the basin becomes larger if frequency is higher. For both horizontal and vertical surface motions, increasing of the frequency tends to cause more complex pattern of the maximum amplitude distribution along the basin surface.

From Fig. 8, we find that the distribution patterns of the maximum surface displacement can be separated into two types. For $\eta$ of 0.75 and 1.0, we see relatively smooth distribution of the maximum surface amplitudes for both horizontal and vertical components. When $\eta$ is larger (2.0, 3.0 and 4.0), the distribution patterns are similar but become quite complex. This is due to the changing of the seismic motion behavior of the basin. For $\eta$ of 0.75 and 1.0, the wavelength of incident wave is 2.67 and 2.0 times 
of the radius of the basin, respectively. Since the wavelength is much longer than the dimension of the basin, the incident wave "ignores" the detailed variations of sediments inside the basin and in consequence the seismic motion of the basin is mainly characterized by the whole basin motion with simple pattern. Bard and Bouchon (1980) and Bard and Gariel (1986) found that for deeper basin like the models in this paper, the prevailing effects are the two-dimensional resonance patterns that are developed by the in phase interference of edge generated surface waves. That is almost similar to the case of low frequency wave incidence model in our simulation.

\section{Discussion and Conclusions}

It has long been recognized that even for the homogeneous basin of low velocity sediment, the duration of ground motion on the basin is much prolonged and the wave amplification in the basin is much enhanced as compared with the bedrock station records. In the basin, the edge-generated Rayleigh wave induced by conversion from the $\mathrm{S}$-wave incidence at the basin edge is a dominant feature for very long time inside the basin after the arrival of major body waves (e.g., Hisada and Yamamoto, 1996). When the impedance contrast is large, the surface waves are reflected at the basin edges and propagate repeatedly between the edges. The same phenomena have also been observed in this paper for the homogeneous case and that is much enhanced for the inhomogeneous cases. The existence of vertical velocity gradient prolongs the duration of the basin motion. The basin-edge generated Rayleigh waves have smaller phase velocity and larger amplitude for greater velocity gradient. In case of $S H$-wave incidence, as mentioned in previous studies (Bard and Gariel, 1986; Benites and Aki, 1994), the amplification of ground motion in the basin is enhanced with large velocity gradient inside the basin. In case of $S V$-wave incidence, our study has found the amplification around the edge area is also observed to be enhanced, especially for the vertical motion. For the frequency values of input signal used in this study, we found that the frequency of incident plane wave obviously affects the distribution pattern of the maximum surface displacement.

Through the parametric simulation study carried out for a semi-cylindrical sediment-filled basin with vertical velocity gradient in case of incident plane $S V$ wave, we found that the vertical velocity gradient has strong effect on the ground motion on a sediment-filled basin. Phenomena that observed in 2D homogeneous sedimentary basin (Bard and Bouchon, 1980) are also observed in this study. But we found that the increase of vertical velocity gradient strongly enhances these phenomena. Vertical velocity gradient makes the distribution of the maximum horizontal amplitude of ground motion more complicated, especially for the early phases that contribute mainly to horizontal motion. For the basin-edge generated Rayleigh waves which contribute mainly to the later time of the vertical motion, increasing vertical velocity gradient makes the phase velocity lower, the duration longer, the amplitude larger and the reflection from the boundary stronger. Vertical velocity gradient generates later phases in the surface motion, so that the surface motion has larger amplitude in later time and is more complicated for greater vertical velocity gradient. Previous studies on response of sedimentary basin subject to plane wave incidence found the edge areas of the basin undergo strong differential motion. In this study we further found the increasing of vertical velocity gradient makes the differential motion stronger, especially for the vertical component. Our results suggest the importance of accurate representation of vertical velocity profile when we construct a structural model for performing realistic simulation of ground motion.

Acknowledgments. The authors would like to thank Y. Hisada for constructive comments and criticism that improved the manuscript. Part of this work was done when the first author (Y.B.W.) joined in the Seismology and Earthquake Engineering Training Course in 1993-1994 in IISEE (International Institute of Seismology and Earthquake Engineering) sponsored by the Japan International Cooperation Agency (JICA). This study was partially supported by a Grant-in-Aid for Scientific Research (No. 08248111) from the Ministry of Education, Science, Sports and Culture of Japan.

\section{Appendix. Validity and Accuracy of the Pseudo- spectral Method}

To check the validity and accuracy of the pseudospectral code used in this paper, we compare synthetic seismograms calculated using the pseudospectral method with those obtained using the discrete wave number method (Bouchon and Aki, 1977). The model we used for the comparison calculation is a half space. The values of density, $P$ - and $S$-wave velocity are the smallest values used in the four models (i.e., the values at the surface of Model 4). The grid spacing is $0.1 \mathrm{~km}$ in both horizontal and vertical directions and the time interval is $0.005 \mathrm{~s}$. All of these values are the same as used in the calculations shown in the previous sections. The source is a double-couple point source with the moment tensor components $M x z=M z x=$ $1.0 \times 10^{12} \mathrm{Nm}$ at a depth of $4 \mathrm{~km}$. Source time function is a Ricker wavelet with peak frequency of $1.0 \mathrm{~Hz}$. The number of grid points per the corresponding shear wavelength is 7.5. Synthetic seismograms at three observation points at the free surface are calculated. The locations of source and the observation points are shown in Fig. A1.

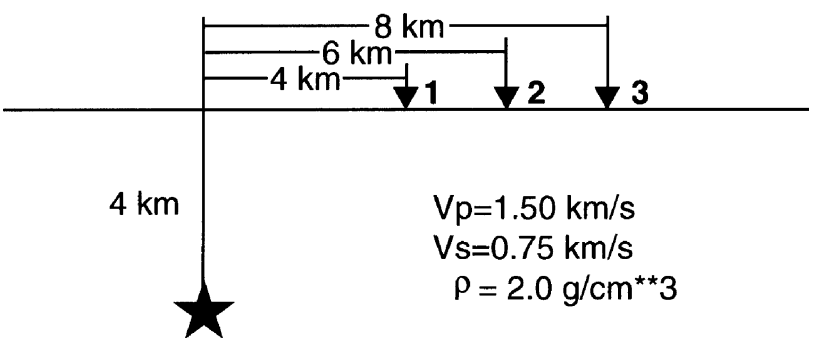

Fig. A1. Half space model used for the comparison calculation. Star is the source position and inverted triangles are the positions of the three observation points at the free surface. 

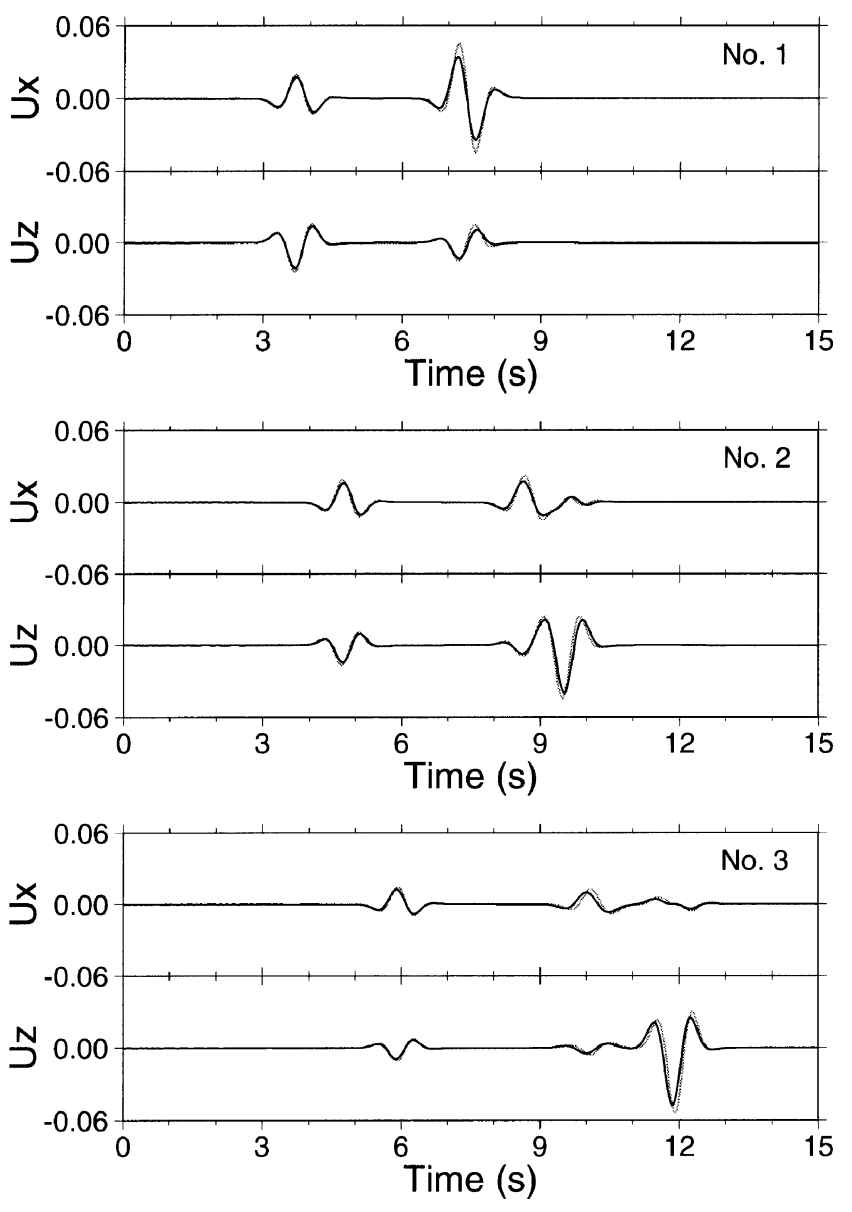

Fig. A2. Comparison of synthetic seismograms at three observation points. Black lines are the results calculated by the pseudospectral method, while grey lines are those calculated by the discrete wave number method.

Figure A2 displays the computed seismograms at the three observation points. At observation point 1 , the direct $P$ and $S$ waves radiated from the source are clearly seen. At observation points 2 and 3 , the $S P$ wave refracted at free surface arrives between the direct $P$ and $S$ waves. The overall agreement between the waveforms obtained by the two methods is good.

\section{References}

Anderson, J. G., P. Bodin, J. N. Brune, J. Prince, S. K. Singh, R. Quaas, and M. Onate, Strong ground motion from the Michoacan, Mexico, earthquake, Science, 233, 1043-1049, 1986.

Bard, P.-Y. and M. Bouchon, The seismic response of sedimentaryfilled valleys, Part II. The case of incident $P$ and $S V$ waves, Bull. Seis. Soc. Am., 70, 1921-1941, 1980.

Bard, P.-Y. and J. Gariel, The seismic response of two-dimensional sedimentary deposits with large vertical velocity gradients, Bull. Seis. Soc. Am., 76, 343-346, 1986.

Benites, R. and K. Aki, Ground motion at mountains and sedimentary basins with vertical seismic velocity gradient, Geophys. J. Int., 116, 95-118, 1994.

Bouchon, M. and K. Aki, Discrete wave-number representation of seismic-source wave fields, Bull. Seis. Soc. Am., 67, 259-277, 1977.

Cerjan, C., D. Kosloff, R. Kosloff, and M. Reshef, A nonreflecting boundary condition for discrete acoustic and elastic wave equations, Geophysics, 50, 705-708, 1985.

Daudt, C., L. Braile, R. Nowack, and C. Chiang, A comparison of finitedifference and Fourier method calculations of synthetic seismogram, Bull. Seis. Soc. Am., 79, 1210-1230, 1989.

Fornberg, B., The pseudospectral method: Comparisons with finite differences for the elastic wave equation, Geophysics, 52, 483-501, 1987.

Furumura, T., H. Takenaka, and K. Kotetsu, Numerical 3-D modeling of seismic waves by the pseudospectral method, Butsuri-Tansa, 49, 536-548, 1996 (in Japanese with English abstract).

Furumura, T., B. L. N. Kennett, and H. Takenaka, Parallel 3-D pseudospectral simulation of seismic wave propagation, Geophysics, 63, 279-288, 1998.

Gao, S., H. Liu, P. M. Davis, and L. Knopoff, Localized amplification of seismic waves and correlation with damage due to the Northridge earthquake: evidence for focusing in Santa Monica, Bull. Seis. Soc. Am., 86, 209-230, 1996.

Graves, R. W., Three-dimensional computer simulations of realistic earthquake ground motions in regions of deep sedimentary basins, in The Effects of Surface Geology on Seismic Motion, edited by K. Irikura et al., 103 pp., Balkema, Rotterdam, 1998.

Haskell, N. A., The dispersion of surface waves on multilayered media, Bull. Seis. Soc. Am., 43, 17-34, 1953.

Hisada, Y. and S. Yamamoto, One-, two-, and three-dimensional site effects in sediment-filled basins, Proceedings of the 11th World Conference on Earthquake Engineering, Paper No. 2040, Acapulco, Mexico, 1996.

Hisada, Y., K. Aki, and T. L. Teng, 3-D simulations of surface wave propagation in the Kanto sedimentary basin, Japan part 2: application of the surface wave BEM, Bull. Seis. Soc. Am., 83, 1700-1720, 1993.

Kawase, H., Effects of sedimentary basins on strong motion-in Mexico city and in Kobe city, in Comprehensive Study of Strong Ground Motion Prediction, edited by K. Irikura, 71 pp., DPRI, Kyoto University, 1996 (in Japanese with English abstract).

Kawase, H., S. Matsushima, R. Graves, and P. Somerville, Threedimensional wave propagation analysis of simple two-dimensional basin structures with special reference to "the basin-edge effect", Zisin (J. Seis. Soc. Japan), 50, 431-449, 1998 (in Japanese with English abstract).

King, J. L. and B. E. Tucker, Observed variations of earthquake motion across a sediment-filled valley, Bull. Seis. Soc. Am., 74, 137-151, 1984.

Kosloff, D. and E. Baysal, Forward modeling by a Fourier method, Geophysics, 47, 1402-1412, 1982.

Kosloff, D., M. Reshef, and D. Loewenthal, Elastic wave calculation by the Fourier method, Bull. Seis. Soc. Am., 74, 875-891, 1984.

Takenaka, H., Computational methods for seismic wave propagation in complex subsurface structures, Zisin (J. Seis. Soc. Japan), 46, 191205, 1993 (in Japanese with English abstract).

Takenaka, H., Y. Wang, and T. Furumura, An efficient approach of the pseudospectral method for modelling of geometrically symmetric seismic wavefield, Earth Planets Space, 51, 73-79, 1999.

Y. Wang (e-mail: wang @geo.kyushu-u.ac.jp), H. Takenaka (e-mail: takenaka@geo.kyushu-u.ac.jp), and T. Furumura (e-mail: furumura@iwa.hokkyodai.ac.jp) 